\section{Die nichtinvasive Beatmung in der Beatmungsentwöhnung}

\section{Beatmungsentwöhnung}

Die invasive maschinelle Beatmung mittels Endotrachealtubus ist ein etabliertes Verfahren in der Behandlung der akuten Ateminsuffizienz. Sie dient der Oxigenierung des Blutes, der $\mathrm{CO}_{2}$-Elimination und schützt die Atemorgane vor weiteren Schäden. Gleichzeitig jedoch sind mit der externen Überdruckbeatmung eine Reihe von unerwünschten Wirkungen und möglichen Komplikationen verknüpft. Neben Druck-/ Volumenschädigungen, die heute weitgehend verhindert werden können, stellt sich bei beatmeten Patienten unvermeidbar eine positive Flüssigkeitsbilanz ein, während die kardiale Auswurfleistung bei gesundem Herzen und bei insuffizientem rechten Herzen unter Beatmung reduziert ist. Auch ist die Perfusion und Sauerstoffaufnahme wichtiger innerer Organe eingeschränkt. Eines der wesentlichen Risiken intubierter und beatmeter Patienten ist die Pneumonie. In einer prospektiven Studie stieg das Pneumonierisiko mit der Beatmungsdauer nahezu linear an und betrug nach 10 Tagen Beatmung 6,5\%, nach 20 Tagen 19\% und nach 30 Tagen 28\% [1]. Die kumulative Prävalenz einer Pneumonie wurde für die ersten 3 Tage unter Beatmung auf 8,5\%, für Tag 7 auf 21,1\%, für Tag 14 auf $32,4 \%$ und für eine Beatmung über mehr als 14 Tage auf $45,6 \%$ berechnet [2]. Aus all diesen Gesichtspunkten ergibt sich die Notwendigkeit, die maschinelle Beatmung so früh wie möglich $\mathrm{zu}$ beenden. Den Prozeß der stufenweisen Reduktion der Beatmungsintensität mit dem Ziel, die Beatmung zu beenden, nennt man Beatmungsentwöhnung.

Die einfachste Form der Beatmungsentwöhnung ist die periodische Diskonnektion des Patienten vom Beatmungsgerät mit zunehmender Dauer solcher Spontanatmungsphasen. Der Patient kann extubiert werden, wenn er über mindestens 30 Minuten ohne Zeichen der Erschöpfung und Verschlechterung der arteriellen Blutgase spontan atmen kann. Mit dieser einfachen Methode können etwa 75\% aller Beatmeten, auch bei Einschluß von Patienten mit bronchopulmonalen Vorerkrankungen, erfolgreich im ersten Versuch entwöhnt werden [3,4]. Eine wichtige Voraussetzung zur erfolgreichen Beatmungsentwöhnung ist die Selektion geeigneter Patienten. Erste Voraussetzung ist, daß der Patient die pulmonale Störung, die zur maschinellen Atemhilfe geführt hat, überwunden hat. Dies bedeutet, daß bei einem maximalen positiven endexspiratorischen Druck von $5 \mathrm{cmH}_{2} \mathrm{O}$ eine arterielle Sauerstoffsättigung von mindestens $90 \%$ bei einem

Pneumologie 2000; 54: 10-15

(C) Georg Thieme Verlag Stuttgart · New York ISSN 0934-8387
J. Lorenz, S. Friesecke, M. Huntemann, C. Kelbel

Abteilung für Pneumologie, Infektiologie, Intensivmedizin und Schlafmedizin, Kreiskrankenhaus Lüdenscheid inspiratorischen Sauerstoffanteil $\left(\mathrm{FiO}_{2}\right)$ von maximal $45 \%$ erreicht wird. Diese Bedingung berücksichtigt allerdings nur die Gasaustauschfunktion der Lunge und nicht die Fähigkeit der Ventilationspumpe, die Atemarbeit zu übernehmen. Weitere, allgemein akzeptierte Voraussetzungen zur erfolgreichen Beatmungsentwöhnung ist die hämodynamische Stabilität des Patienten (Ausschluß einer myokardialen Ischämie, einer Schock-Konstellation oder neu aufgetretener Arrhythmien), der Ausschluß einer neu aufgetretenen Infektion und einer offensichtlichen zentral-nervösen Dysfunktion. Da die oben genannte Erfolgsrate von $75 \%$ auch dann nicht übertroffen wird, wenn all diese Regeln beachtet werden, sind in neuerer Zeit zahlreiche Untersuchungen durchgeführt worden, um geeignete Entwöhnungskandidaten prospektiv identifizieren zu können. Dabei wurden Gasaustauschparameter, Parameter der Atemmuskelkraft und -reserven, Parameter der Atemarbeit sowie kombinierte Parameter geprüft [5]. Allein die große Anzahl der vorgeschlagenen Entwöhnungsdeterminanten zeigt, daß keiner dieser Parameter befriedigen konnte. Die Beurteilung der Entwöhnbarkeit Langzeitbeatmeter, insbesondere bei Vorliegen kardiopulmonaler Vorerkrankungen, erfordert eine komplexe Synopsis, die in erster Linie klinische Erfahrung benötigt. Diejenigen Patienten, die nach wiederholten Spontanatmungsversuchen nicht erfolgreich extubiert werden können, benötigen eine Entwöhnungsstrategie. Alle derzeit praktizierten Entwöhnungsstrategien beruhen auf dem Prinzip, den Anteil der Atemarbeit, die vom Patienten zu leisten ist, im Verhältnis zur Gesamtatemarbeit kontinuierlich zu erhöhen, bis der Patient zur Spontanatmung fähig ist. Das Verfahren der synchronisierten intermittierenden mandatorischen Ventilation (SIMV) stützt sich zum Beispiel auf die kontinuierliche Rücknahme des Anteils maschinell übernommener Atemzüge. Andere Verfahren reduzieren die Atemarbeit durch eine inspiratorische Druckunterstützung jeden Atemzuges („pressure support ventilation“, PSV), wobei die Entwöhnung durch schrittweise Reduktion der Druckunterstützung umgesetzt wird [6]. Systematische Beobachtungen zeigen, daß es mit Hilfe solcher Entwöhnungsstrategien gelingt, etwa $80 \%$ der Patienten, die durch periodische Spontanatmungsversuche nicht entwöhnt werden können, letztlich der Extubation zuzuführen [3,4]. Somit gilt etwa einer von 20 Patienten, die bei Vorliegen chronisch kardiopulmonaler oder neuromuskulärer Vorerkrankungen beatmet werden müssen, als nicht entwöhnbar. Die Durchführung der beschriebenen Entwöhnungsstrategien impliziert einen erheblichen Aufwand an Mühen, Personaleinsatz, Zeit und ökonomischen Ressourcen. Dies wird deutlich, wenn man bedenkt, daß mehr als $40 \%$ der Zeit, in der der Patient vom 
Beatmungsgerät abhängig ist, der Entwöhnung gewidmet werden muß [7].

\section{Erfolglose Beatmungsentwöhnung}

Patienten, bei denen ein Entwöhnungsversuch fehlschlägt, zeigen klinische Zeichen der Ateminsuffizienz und hämodynamische sowie psychovegetative Symptome. Bei einem Spontanatmungsversuch können sich bereits innerhalb weniger Minuten Zeichen der Intolerenz einstellen. Es entwickelt sich eine hochfrequente, oberflächliche Atmung („rapid shallow breathing“) mit einer Atemfrequenz von über 35/min, der arterielle Blutdruck steigt oder fällt um $20 \%$ oder auf Absolutwerte über 180 oder unter $90 \mathrm{~mm} \mathrm{Hg}$ systolisch. Die Herzfrequenz steigt oder fällt um mehr als $20 \%$, die Sauerstoffsättigung fällt auf unter $90 \% \mathrm{ab}$ und meist steigt parallel dazu der arterielle Kohlensäurepartialdruck um mehr als $5 \mathrm{~mm} \mathrm{Hg}$ an, gefolgt von einem Abfall des arteriellen Blut-pH-Wertes auf unter 7,3. Andere Zeichen der Spontanatmungsintoleranz sind das Auftreten kardialer Arrhythmien, der Gebrauch akzessorischer Atemmuskeln, asynchrone Atembewegungen von Thorax und Abdomen, Schweißneigung oder, soweit der Patient dazu in der Lage ist, Angaben über Luftnot $[3,4,8]$. Das beschriebene klinische Bild ist das der akuten Atemmuskelerschöpfung. Ein ganzes Bündel von möglichen Ursachen kann dafür verantwortlich sein. Allgemein gesprochen kann der Patient, der erfolglos vom Respirator entwöhnt wird, die geforderte Atemarbeit nicht leisten. Eine mögliche Ursache ist die intolerable Erhöhung der geforderten Atemarbeit durch gestiegene resistive oder elastische Widerstände oder aber eine Inkompetenz der Atemmuskelpumpe einschließlich des Atemantriebs, der neuromuskulären Kopplung sowie der Kraftübertragung am Thorax während der Inspiration. Ein Teil dieser Einflüsse sind Ausdruck zugrundeliegender kardiopulmonaler Vorerkrankungen oder noch Folgen der akuten respiratorischen Insuffizienz während andere Einflüsse mittelbar oder unmittelbare Folgen der maschinellen Beatmung darstellen.

Ein alveoläres oder interstitielles Lungenödem, ein pneumonisches Infiltrat, Pleuraergüsse oder das Vorliegen eines intrinsischen positiv endexspiratorischen Druckes (PEEPi) oder pulmonale Atelektasen können ebenso verantwortlich sein für einen erhöhten Bedarf an Atemarbeit wie stenosierend wirkendes Bronchialsekret, ein Bronchospasmus oder ein bronchiales Schleimhautödem. Auch auf Einflüsse einer Adipositas ist, vor allem beim flachliegenden Patienten, zu achten. Noch umfangreicher ist die Liste an Möglichkeiten, die zu einer Einschränkung der Kompetenz der Atempumpe führen können. Der Atemantrieb kann eingeschränkt sein durch die fortdauernde Wirkung von Analgosedativa, eine metabolische Alkalose, eine toxische metabolische Enzephalopathie, oder eine Schlafdeprivation, wie sie bei kritisch Kranken häufig beobachtet wird. Die neuromuskuläre Kopplung kann gestört sein durch Medikamente (Muskelrelaxantien, Benzodiazepine, Aminoglykoside) oder durch eine Polyneuropathie bzw. Myopathie, wie sie bei kritisch Kranken häufig, insbesondere bei Anwendung von Glukokortikosteroiden, vorkommen. Malnutrition, Elektrolytstörungen, septische Stoffwechselfolgen oder Inaktivitätsatrophie schwächen das Kontraktionsverhalten der Atemmuskulatur. Die Gesamtenergiebilanz des kritisch Kranken ist negativ und die Speicher an energiereichen Substraten in der Muskelzelle entleert.
Durch akute Krankheitszustände wie Sepsis und Schock ist die Verwertung von Sauerstoff und Energiesubstraten in der Zelle beeinträchtigt. Negative Randbedingungen können das Mißverhältnis zwischen Energieerfordernis und Energiebereitstellung bei der Atmung zusätzlich ungünstig verändern. Dazu zählt die flache Lagerung des Patienten, Fieber, Schmerzen, Angst und Agitation.

Auch die maschinelle Ventilation selbst kann zur Erschöpfung beitragen. Der Endotrachealtubus vergrößert den anatomischen Totraum. Dies spielt insbesondere bei kleinen Atemzugvolumina eine erhebliche Rolle. Der Innendurchmesser des Endotrachealtubus hat etwa den halben Durchmesser der Trachea eines Erwachsenen und erhöht den Atemwegswiderstand damit um etwa das 4 fache. Dies erhöht den benötigten Inspirationsdruck und damit die Atemarbeit erheblich. Es konnte gezeigt werden, daß bei Patienten mit COPD eine inspiratorische Druckunterstützung von bis zu $20 \mathrm{cmH}_{2} \mathrm{O}$ benötigt wird, um den erhöhten Tubuswiderstand zu kompensieren [9]. Das Tubuslumen ist oft bei länger liegenden Tuben durch Inkrustationen und Sekret zusätzlich stenosiert. Benzodiazepine und andere Sedativa werden häufig zum Erzielen einer Toleranz gegenüber dem liegenden Endotrachealtubus verwendet. Sie reduzieren den Atemantrieb und sind muskelrelaxierend. Der liegende Endotrachealtubus und exspiratorische Widerstände seitens des Beatmungsgerätes machen einen effektiven Hustenstoß unmöglich. Dies führt zur Sekretakkumulation mit weiterer Ventilationsbehinderung. Zur kontrollierten Beatmung und vor allem während der Entwöhnung werden häufig Beatmungsverfahren gewählt, bei denen der Patient die Inspiration triggert, während der Ventilator die so begonnene Inspiration komplettiert („assistierte Beatmung“). Diese Beatmungsform hat das Ziel, das Wohlbefinden des Patienten zu erhöhen und die Atemarbeit zu reduzieren. Direkte Untersuchungen des Energieverbrauchs unter verschiedenen Beatmungsformen und Berechnungen der Atemarbeit zeigten jedoch, daß die „Triggerarbeit“ erheblich ist und in manchen Fällen ein Energieverbrauch wie bei Spontanatmung resultiert $[10,11]$. Bei schlechter Patienten-Ventilatorkoordination kann die Atemarbeit noch weiter erheblich ansteigen. Das Verhältnis der erbrachten Atemarbeit des Patienten unter assistierter Beatmung im Verhältnis zu seiner maximal erbringbaren Atemarbeit ist im Einzelfall nicht erkennbar und erst bei beginnender Erschöpfungssymptomatik zu verifizieren. Letztlich fragt sich auch, ob die Entwöhnungsstrategie der langsam zunehmenden Dauerbelastung der Ventilationspumpe unter assistierter Beatmung ein geeignetes Trainingskonzept für den Patienten darstellt. Sportmedizinische Erfahrungen zeigen jedenfalls, daß biochemische und morphologische Trainingsvorgänge am quergestreiften Muskel durch Intervallbelastungen günstiger zu beeinflussen sind als durch zunehmende Dauerbelastung. Neuere Studien zum Vergleich verschiedener Entwöhnungsverfahren (intermittierende Spontanatmung, SIMV, PSV) haben folgendes ergeben: Der SIMV-Modus ist den anderen beiden Verfahren unterlegen und die druckunterstützte Spontanatmung erzielt nicht eindeutig bessere Ergebnisse als intermittierende Spontanatmungsversuche $[3,4]$. Insgesamt zeigt sich, daß der intubierte Patient in der Entwöhnungsphase einer erheblichen Belastung seiner Atempumpe unterliegt, die zum Teil durch die gewählte invasive Beatmung selbst bedingt ist und damit der Erfolg des Entwöhnungsversuchs in Frage gestellt wird. 
Muß ein Patient nach zunächst erfolgreicher Entwöhnung und Extubation erneut reintubiert und maschinell beatmet werden, so ist dies prognostisch ungünstig. Das Risiko einer Reintubation beträgt im Mittel 15\%. Eine Reintubation erhöht das Risiko einer nosokomialen Pneumonie und verlängert die Aufenthaltsdauer auf der Intensivstation [12]. In einer Untersuchung betrug die Letalität von erfolgreich extubierten Patienten 12\%, bei Mißerfolg jedoch 43\% [13]. Diese Befunde sprechen gegen die fortgesetzte Verwendung eines Endotrachealtubus während des Entwöhnungsvorganges und im Fall einer erfolglosen Extubation.

\section{Prinzip und Ergebnisse der nichtinvasiven Beatmungsentwöhnung}

Die nichtinvasive Beatmung über eine meist nasale Maske ist ein Verfahren, das sich im Langzeitsupport von Patienten mit chronischer Ateminsuffizienz bei erschöpfter Atempumpe bewährt hat. In der Behandlung der akuten, hyperkapnischen Ateminsuffizienz auf der Intensivstation sind zahlreiche positive Erfahrungen gewonnen worden [14-16]. Auch bei der Behandlung der akuten respiratorischen Insuffizienz liegen günstige Erfahrungen vor $[17,18]$. Es liegt also nahe, den Stellenwert der nichtinvasiven Beatmung auch in der Beatmungsentwöhnung zu bestimmen. Die Methode bietet im Vergleich zur invasiven Beatmung eine Reihe von Merkmalen, die in der Entwöhnung genutzt werden können: Bei richtiger Durchführung benötigt der Patient keine Sedierung, da der Endotrachealtubus entfällt. Alle oben geschilderten, mit dem Endotrachealtubus verbundenen unerwünschten Effekte und Komplikationen spielen keine Rolle. Bei Verwendung einer nasalen Maske mit intermittierender Verwendung ist der Hustenreflex nicht beeinträchtigt. Auch der Schluckreflex ist nicht behindert, so daß eine orale Nahrungsaufnahme möglich ist. Nasale Masken erlauben aber auch die Verwendung nasaler Ernährungssonden ohne wesentliche Maskenundichtigkeit. Eine weiterer Vorteil ist die freie Wählbarkeit der Körperlage des nicht sedierten Patienten. So kann in sitzender Position die Schwerkraft bei der Zwerchfellatmung ausgenutzt werden und bei adipösen Patienten das Zwerchfell tiefer treten. Lungenatelektasen lassen sich bei geschädigtem Parenchym in dieser Position auflösen [19]. Ein weiterer Gesichtspunkt ist die jederzeitige Reversibilität dieser Beatmungsform. Beginn und Ende eines Beatmungsintervalls sind frei wählbar und können jederzeit umgesetzt werden. Auch ist die Kommunizierbarkeit mit dem nicht sedierten Patienten jederzeit möglich, so daß differenzierte Informationen über den klinischen Zustand zu erhalten sind und die psychosoziale Betreuung erheblich erleichtert wird. Ein positiver Begleiteffekt bei Vorliegen eines obstruktiven SchlafapnoeSyndroms oder bei noch schwacher Muskelspannung ist die pneumatische Schienung der oberen Atemwege durch den nasal applizierten Überdruck. Damit werden andere Maßnahmen zur Schienung der oberen Atemwege, zum Beispiel der Wendel-Tubus, überflüssig.

Was sind die Grenzen der Methode? Die Kopplung zwischen Beatmungsgerät und Patient ist bei der Beatmung via Maske wesentlich delikater als bei Verwendung eines Trachealtubus. Die wesentlichen Vorteile der Technik kommen vor allem bei Verwendung nasaler Masken zum Tragen, womit Kompromisse im Hinblick auf orale Luftlecks bei der Erreichung eines vorgewählten endinspiratorischen Volumen- oder Druckziels eingegangen werden müssen. In vielen Fällen ist dieses Luftleck jedoch nicht erheblich oder kann über technische Hilfsmittel (z.B. Kinngurte) reduziert werden. In jedem Fall ist auf eine optimale Anpassung der Maske an die Gesichtsanatomie zu achten. Anderenfalls sind Luftlecks am Maskenrand, meist im Bereich der Nasenwurzel mit Reizung der Augen oder aber Druckstellen, meist im Bereich des Nasenrückens, die Folge. Nasale Druckstellen können innerhalb von wenigen Stunden zur Nekrose führen und das gesamte nichtinvasive Beatmungskonzept in Frage stellen. Aus diesem Grund ist die Bereitstellung zahlreicher Maskenvarianten und -größen auf der Intensivstation, besser noch ein rascher Zugriff auf individuell modellierte Masken, notwendig. Die psychische Toleranz der Maskenatmung stellt in der Entwöhnungsphase selten ein Problem dar. In Einzelfällen jedoch kommen klaustrophobe Reaktionen vor. Ein wesentlicher Gesichtspunkt ist der nach Entfernung des Endotrachealtubus fehlende Schutz gegenüber einer Aspiration, insbesondere im Zusammenhang mit einer gelegentlich vorkommenden Überblähung des Magens im Rahmen der Maskenbeatmung. Vor Beginn der nichtinvasiven Beatmung ist daher in jedem Fall das Aspirationsrisiko zu überdenken und die Schutzreflexe des Patienten zu prüfen. Die Aspirationsgefahr ist bei Verwendung von Nasen/Mundmasken ungleich größer als bei Nasenmasken. Hygienemaßnahmen der oberen Atemwege sind unter der Maskenbeatmung gegenüber der endotrachealen Intubation nur wenig behindert und auch die Durchführung einer gezielten bronchoskopischen Bronchialtoilette unter fortlaufender Beatmung über oral/nasale Maske mit Endoskopieöffnung ist jederzeit möglich. Ein entscheidender Gesichtspunkt bei der Auswahl des nichtinvasiven Verfahrens in der Beatmungsentwöhnung ist die Beachtung von Kontraindikationen [16,20-22]. Dazu zählen ein hohes Aspirationsrisiko oder eine ausgeprägte bronchiale Hypersekretion, die eine regelmäßige endotracheale Absaugung notwendig macht. Problematisch ist die nichtinvasive Beatmung auch bei Vorliegen einer stark wechselnden oder instabilen Atemmechanik, wie sie etwa bei Asthma bronchiale in der Exazerbation vorkommt. Ungünstig für die Methode ist auch eine instabile Hämodynamik mit stark wechselnden Blutdruckwerten und das Vorliegen einer schweren Azidose, zum Beispiel in Verbindung mit einer ausgeprägten Hyperkapnie. Patienten mit Koma oder Verwirrtheitszuständen sind ebenfalls von dem Verfahren auszuschließen, da die Kooperation des Patienten vorausgesetzt werden muß. Auch eine stark veränderte Gesichtsanatomie oder Mittelgesichtsverletzungen können die Anwendung der Methode im Einzelfall unmöglich machen. Außerdem gelten die allgemeinen Voraussetzungen zum Entwöhnungsversuch bei intubierten Patienten auch für die nichtinvasive Form der Entwöhnung. Das Monitoring des mittels Maskenatmung zu entwöhnenden Patienten ist umfassender als beim intubierten Patienten und bezieht sich nicht nur auf die Vitalparameter (Puls, Blutdruck, Sauerstoffsättigung, Blutgasanalyse), sondern erweitert sich auf die engmaschige Beurteilung des Bewußtseinszustandes, des Hustenreflexes, der bronchialen Sekretion, der Auskultationsgeräusche, des Atemmusters (Atemtiefe und Frequenz), der Kooperation zwischen Patient und Ventilator sowie auf den psychischen Zustand des Patienten. Dies impliziert, daß zu der intensivmedizinisch-technischen Ausstattung auch die intensive pflegerische und ärztliche Betreuung hinzutreten muß. Als Regel gilt, daß die ständige Anwesenheit einer 
Tab. 1 Studien zur Beatmungsentwöhnung mit nichtinvasiver maschineller Ventilation

\begin{tabular}{llllll}
\hline Referenz & Studientyp & $\begin{array}{l}\text { Patientenzahl } \\
(\mathrm{n})\end{array}$ & $\begin{array}{l}\text { Erfolgsrate } \\
(\%)\end{array}$ & $\begin{array}{l}\text { Teilentwöhnung } \\
(\%)\end{array}$ & $\begin{array}{l}\text { Letalität } \\
(\%)\end{array}$ \\
\hline Laier-Groeneveld, 1992 [23] & retrospektiv & 35 & 94 & 83 & 6 \\
Udwadia, 1992 [24] & retrospektiv & 22 & 80 & 80 & n.a. \\
Restrick, 1993 [25] & retrospektiv & 14 & 92 & 14 & 7 \\
Nava, 1998 [26] & prospektiv ${ }^{1}$ & 25 & 88 & n.a. & 8 \\
Girault, 1999 [27] & prospektiv & 17 & 76 & 41 & 0 \\
\hline
\end{tabular}

${ }^{1}$ Teilkollektiv einer randomisierten, kontrollierten Studie

erfahrenen Pflegeperson notwendig ist und der betreuende Arzt jederzeit hinzugerufen werden können muß.

Mehrere klinische Untersuchungen haben sich mit der Durchführbarkeit und den Ergebnissen der Beatmungsentwöhnung durch nichtinvasive Ventilation befaßt (Tab.1). Die Mehrzahl dieser Untersuchungen sind retrospektive Auswertungen von Patientenserien, bei denen im Rahmen einer schwierigen Beatmungsentwöhnung die nichtinvasive Beatmung als Ultima ratio eingesetzt wurde. 1992 berichteten Laier-Groeneveld und Crieé über 35 Patienten mit chronischer Ateminsuffizienz im Rahmen chronisch-obstruktiver Atemwegserkrankungen, neuromuskulären Erkrankungen und Thoraxdeformitäten, die im Mittel nach 64 Tagen maschineller Beatmung als nicht entwöhnbar galten. Alle Patienten waren hyperkapnisch und multiple Spontanatmungsversuche waren fehlgeschlagen. Die intermittierende Durchführung der maschinellen Ventilation via Nasenmaske mit volumengesteuerten Ventilatoren führte zur Normalisierung der Blutgase. Unter dieser Entlastung der Atempumpe verbesserte sich die Spontanatmungstoleranz erheblich. Bei fast allen Patienten konnten Tracheostomata verschlossen werden. 94\% der Patienten konnten nach einer mittleren Behandlungsdauer von 26 Tagen stationär entlassen werden. Die überwiegende Mehrzahl verblieb jedoch mit einer nächtlichen Beatmungsunterstützung im Sinne einer partiellen Beatmungsentwöhnung. Bemerkenswert war, daß von den erfolgreich behandelten Patienten noch 97\% nach einem Jahr, 92\% nach 2 Jahren und 89\% nach fünf Jahren lebten. Dies bedeutet, daß der Übergang in ein Heimbeatmungsprogramm bei partiell entwöhnbaren Patienten mit einer recht guten mittelfristigen Prognose verbunden ist [23]. Über ähnliche Ergebnisse berichten Udwadia [24] und Restrick [25]. Auch hier wurden Patienten mit chronischer Kompromittierung der Atempumpe auf zentraler oder neuromuskulärer Ebene oder im Rahmen einer chronisch-obstruktiven Atemwegserkrankung mit schwieriger Beatmungsentwöhnung untersucht. 80-90\% der Patienten konnten erfolgreich mittels nasaler intermittierender Ventilation entwöhnt werden, wobei auch hier ein wechselnder Anteil der Patienten nur partiell entwöhnt werden konnte. Bei allen Untersuchungen lag, soweit angegeben, die Letalität bei unter $10 \%$ der schwer erkrankten Patienten. 1998 wurde von Nava und Mitarb. eine erste prospektive Untersuchung vorgelegt [26]. 68 Patienten mit chronisch obstruktiver Atemwegserkrankung, von denen jedoch nur 50 Patienten randomisiert werden konnten, wurden nach erfolglosem Entwöhnungsversuch mit liegendem Endotrachealtubus mit intermittierendem Spontanatmungsversuch entweder extubiert und der nichtinvasiven Beatmung mittels nasaler Maske zugeführt oder aber unter weiterer Intubation mit inspiratorischer
Druckunterstützung (PSV) entwöhnt. Die Studie erfolgte multizentrisch in drei Intensivtherapiestationen und die Behandlungsgruppen wurden randomisiert zugeteilt. Es zeigte sich, daß mit allen untersuchten Zielvariablen mit der nichtinvasiven Entwöhnungsmethode bessere Ergebnisse zu erzielen waren. Die mittlere Beatmungsdauer war mit 10,2 versus 16,6 Tagen kürzer, die Letalität mit $8 \%$ versus $28 \%$ geringer und der Entwöhnungserfolg mit $88 \%$ versus $68 \%$ günstiger. Ein entscheidender Faktor für die guten Ergebnisse der nichtinvasiven Methode war das fehlende Auftreten von nosokomialen Pneumonien, während 28\% der weiterhin intubierten Patienten mit PSV-Support Pneumonien entwikkelten. Beide Patientengruppen waren hinsichtlich der Ausgangsbedingungen vergleichbar. Eine französische unizentrische Untersuchung verglich ebenfalls prospektiv randomisiert PSV unter Intubation mit der nasalen Atemhilfe nach erfolglosen Spontanatemversuchen [27]. Der Entwöhnungserfolg war in beiden Gruppen mit etwa 75\% gleich. Durch das nichtinvasive Verfahren konnte die Intubationsdauer verkürzt werden, während die Dauer der Entwöhnungsphase verlängert war. Die Anzahl von Komplikationen während der Beatmung war in der Gruppe der intubierten Patienten deutlich, aber nicht signifikant, erhöht (neun von 16 versus sechs von 17 Patienten). Die Aufenthaltsdauer auf der Intensivstation und im Krankenhaus und die Vierteljahresletalität waren in beiden Gruppen gleich. Die Vorteile der nichtinvasiven Entwöhnung waren in dieser Untersuchung nicht so deutlich ausgeprägt, wenngleich ein zumindest gleich großer Entwöhnungserfolg bei kürzerer Intubationsphase - und damit geringerer Komplikationsrate - erzielt werden konnte. Bemerkenswert war, daß partielle Entwöhnungen mit anschließender Heimbeatmung nur in der Gruppe mit nichtinvasiver Beatmung - und hier bei $41 \%$ der Patienten vorkamen. Aufgrund der vorgelegten Studien kann bisher nicht die Frage beantwortet werden, ob Patienten ohne spezifische Probleme der Atempumpe von der frühzeitigen Extubation eben so sehr profitieren. Neuere Untersuchungen zur nichtinvasiven Beatmung bei akuter Ateminsuffizienz zeigen immerhin, daß auch hier die Vermeidung von nosokomialen Pneumonien einen wesentlichen Anteil am Gesamterfolg hat $[28,29]$.

\section{Praktische Durchführung}

Für die nichtinvasive Beatmungsentwöhnung gelten die allgemein akzeptierten Bedingungen für den Entwöhnungsbeginn. Die zur Beatmung führende Akuterkrankung sollte kontrolliert sein und eine schwere respiratorische Insuffizienz (Sauerstoffsättigung unter $90 \%$ bei einem $\mathrm{FiO}_{2}$ von 0,45 und einem PEEP von $5 \mathrm{cmH}_{2} \mathrm{O}$ ) sollte nicht mehr vorliegen. Auch 
interkurrente Erkrankungen (Infektionen, dekompensierte Herzinsuffizienz) sollten ausgeschlossen sein. Dagegen ist es nicht notwendig, daß konventionelle Extubationskriterien gegeben sind, da die nichtinvasive Beatmung eine ausreichende Ventilation gewährt. Zwei weitere wichtige Voraussetzungen sind jedoch die Kooperationsfähigkeit des Patienten (Ausschluß von Koma, Verwirrtheitszuständen) und die Fähigkeit, zumindest für 10-30 Minuten ohne Atemunterstützung spontan zu atmen. Die Erfolgsaussichten der nichtinvasiven Beatmungsentwöhnung sind um so größer, je wacher und kooperationsbereiter der Patient ist [30]. Die Beatmung kann mit konventionellen Geräten (z. B. EVITA, Firma Draeger) oder aber mit einfacheren druck- oder volumengesteuerten Heimbeatmungsgeräten durchgeführt werden. Die Auswahl einer Drucksteuerung versus Volumensteuerung hängt ab von dem zuvor gewählten Beatmungsmodus, der Grunderkrankung und dem subjektiven Empfinden des Patienten. Grundsätzlich sind beide Beatmungsformen möglich. Auf jeden Fall sollte das Atemminutenvolumen so gewählt werden, daß Normokapnie oder allenfalls eine leichte Hyperkapnie erzielt wird. Die zusätzliche Gabe von Sauerstoff ist meist notwendig und orientiert sich an der arteriellen Sauerstoffsättigung, die über $90 \%$ betragen sollte. Bei Patienten mit obstruktiver Atemwegserkrankung sollte auf das Vorliegen eines PEEPi geachtet werden. Dieses Phänomen erhöht die Atemarbeit, da zum Triggern des Gerätes sowohl ein initialer Druckabfall (oder Strömungsanstieg) sowie die Überwindung des PEEPi notwendig ist. Daher sollte bei Vorliegen eines PEEPi ein extrinsischer PEEP gewählt werden, der etwas unterhalb des intrinsischen Wertes liegt [31]. Besondere Aufmerksamkeit ist dem Patienten in den ersten Stunden der nasalen Ventilation zu widmen. Dies bezieht sich vor allem auf die kardiopulmonale Situation, den Patientenkomfort sowie Sitz und Anpassung der nasalen Maske, vor allem im Hinblick auf Luftlecks und sich entwickelnde Druckstellen. In dieser Phase müssen Masken häufig ausgewechselt werden. Eine halb sitzende Körperposition des Patienten ist häufig angenehm und verbessert die Ventilation.

Von der nichtinvasiven Ventilation sind Patienten auszuschließen, die sich bereits zu Beginn der Entwöhnung in einer erschöpften Situation befinden. In diesem Fall sollte zunächst für 24 bis 48 Stunden eine kontrollierte Beatmungsphase vorangeschaltet werden, um die muskulären Energiespeicher zu füllen. Begleitend ist auf Hinweise auf das Vorliegen eines fortgeschrittenen Katabolismus zu achten (hoher Serum- und Urinharnstoff). Frühzeitig ist die Ernährungsbehandlung entsprechend anzupassen.

Eine entscheidende Frage ist die Auswahl von geeigneten Patienten für die nichtinvasive Beatmungsentwöhnung. In Anbetracht der Tatsache, daß die überwiegende Mehrzahl der Patienten mit intermittierenden Spontanatmungsversuchen erfolgreich entwöhnt werden kann, sollte ein derartiger Entwöhnungsversuch zunächst vorangeschaltet werden. Falls dies aufgrund einer raschen Patientenermüdung mißlingt, insbesondere bei Vorliegen von Erkrankungen, die die Atemmuskelpumpe kompromittieren, ist ein Entwöhnungsversuch mittels nasaler Ventilation indiziert. Geeignete Kandidaten sind auch Patienten mit Atemmuskelermüdung nach Extubation. Hier kann eine Reintubation vermieden werden. In dieser Situation ist die Entscheidung jedoch möglichst frühzeitig zu treffen, da ein fortgeschrittener Erschöpfungszu- stand (Nasenflügeln, Atemfrequenz über 35/min) die Erfolgsaussichten verschlechtert.

Die nichtinvasive Beatmung sollte in der ersten Phase so lange fortgeführt werden, bis der Patient frei von Luftnot ist, die Blutgase sich deutlich verbessern und die Maskenanpassung abgeschlossen ist. Im Anschluß daran kann mit Spontanatmungsversuchen unter nasaler $\mathrm{O}_{2}$-Gabe von zunächst kurzer Dauer (10-30 Minuten) begonnen werden. Diese Versuche sind vor der starken Ausprägung von Erschöpfungssymptomen abzuschließen. Unter Beibehaltung eines normalen Tag/ Nacht-Rhythmus kann die Dauer und Frequenz der Spontanatmungsversuche langsam, nach den Möglichkeiten des Patienten, erhöht werden. In dieser Phase ist absehbar, ob eine komplette oder partielle Entwöhnung vom Ventilator möglich ist. Bei lediglich partieller Beatmungsentwöhnung sind Maßnahmen zur Einschleusung des Patienten in ein Heimbeatmungsprogramm zu treffen.

Die nichtinvasive Beatmung mittels nasaler Maske hat sich als ein durchführbares Verfahren zur Beatmungsentwöhnung von schwierig entwöhnbaren Patienten erwiesen. Die Indikation ist vor allem bei Patienten mit chronischer Kompromittierung der Atempumpe, insbesondere bei chronisch-obstruktiven Atemwegserkrankungen, gegeben. Vor Etablierung der Methode als Alternativverfahren zu kontinuierlichen Entwöhnungstechniken (PSV, SIMV) sind weitere multizentrische, prospektive, kontrollierte Studien notwendig. Nach den bisherigen Ergebnissen liegen die Hauptvorteile in der Prophylaxe nosokomialer Pneumonien und anderer tubusassoziierter Komplikationen sowie in der Vermeidung der mit dem Tubus assoziierten erhöhten Atemarbeit.

\section{Literatur}

${ }^{1}$ Fagon JY, Chastre J, Domart Y, Truvillet JL, Pierre J, Darne C, Gibert C. Nosocomial pneumonie in patients receiving continuous mechanical ventilation: Prospective analysis of 52 episodes with use of a prospective specimen brush and quantitative culture techniques. Am Rev Respir. Dis 1989; 139: 877 - 884

${ }^{2}$ Ruiz-Santana S, Garcia A, Esteban A, Guerra L, Alvarez B, Corcia S, Gudin J, Martinez A, Quintana E, Armengol S, Gregori J, Arenzana A, Rosado L, Sanmartin A. ICU pneumonias: A multiinstitutional study. Crit Care Med 1987; 15: 930 - 932

${ }^{3}$ Brochard L, Rauss A, Benito S, Conti G, Mancebo J, Rekik N, Gasparetto A, Lemaire F. Comparison of three methods of gradual with drawal from ventilatory support during weaning from mechanical ventilation. Am J Respir Crit Care Med 1994; 150: 896 - 903

${ }^{4}$ Esteban A, Alia I, Gordo F, Fernandez R, Solsona JF, Vallverdu I, Macias S, Allegue JM, Blanco J, Carriedo D, Leon M, De la Cal MA, Taboada F, Gonzalez de Veazco J, Palazon E, Carrizosa F, Tomas R, Suarez J, Goldwasser R for the Spanish Lung Failure Collaborative Group. Extubation outcome after spontanous breathing trials with T-tube or pressure support ventilation. Am J Respir Crit Care Med 1997; 156: 459 - 465

${ }^{5}$ Vassukajopoulos T, Zakynthinos S, Roussos C. The conventional approach to weaning from mechanical ventilation. Eur Respir Mon 1998; 8: 266 - 298

${ }^{6}$ Esteban A, Alia I. Clinical management of weaning from mechanical ventilation. Intensive Care Med 1998; 24: 999 - 1008

${ }^{7}$ Esteban A, Alia I, Ibanez J, Benito S, Tobin MJ and the Spanish Lung Failure Collaborative Group. Modes of mechanical ventila- 
tion and weaning. A national survey of Spanish hospitals. Chest 1994; 106: 1188 - 1193

${ }^{8}$ Ely EW, Baker AM, Dunagan DP, Burke HL, Smith AC, Kelly PT, Johnson MM, Browder RW, Bowton DL, Haponik EF. Effect on the duration of mechnical ventilation of identifying patients capable of breathing spontanously. N Engl J Med 1996; 335: 1864 - 1869

${ }^{9}$ Brochard L, Rua F, Lorino H, Lemaire F, Harf A. Inspiratory pressure support compensates for the additional work of breathing caused by the endotracheal tube. Anaesthesiology 1991; 75: $735-745$

${ }^{10}$ Rasche K, Laier-Groeneveld G, Weyland W, Braun U, Hüttemann U, Criee C-P. Sauerstoffverbrauch der Atemmuskulatur unter kontrollierter bzw. assistierter Beatmung bei Patienten mit chronischer Ateminsuffizienz. Med Klin 1994; 89: 43 - 46

${ }^{11}$ Ward ME, Corbeil C, Gibbons W, Newman S, Macklem PT. Optimisation of respiratory muscle relaxation during mechanical ventilation. Anaesthesiology 1988; 69: 29 - 35

${ }^{12}$ Craven DE, Steger KA. Epidemiology of nosocomial pneumonia. New perspectives on an old disease. Chest 1995; 108: 1S - 16S

13 Epstein SK, Ciubotaru RL, Wong JB. Effect of failed extubation on the outcome of mechanical ventilation. Chest 1997; 112: $186-$ 192

${ }^{14}$ Brochard L, Isabey D, Piquet J, Amaro P, Mancebo J, Messadi A-A, Brun-Buisson C, Rauss A, Lemaire F, Harf A. Reversal of acute exacerbations of chronic obstructive lung disease by inspiratory assistance with a face mask. N Engl J Med 1990; 323: 1523 1530

15 Meduri GU, Turner RE, Abou-Shala N, Wunderinck R, Tolley E. Noninvasive positive pressure ventilation via face mask. First line intervention in patients with acute hypercapnic and hypoxemic respiratory failure. Chest 1996; 109: 179 - 193

${ }^{16}$ Brochard L, Mancebo J, Wysocki M, Lafoso F, Conti G, Rauss A, Simmoneau G, Benito S, Gasparetto A, Lemaire F, Isabey D, Harf A. Noninvasive ventilation for acute exacerbations of chronic obstructive pulmonary disease. N Engl J Med 1995; 333: 817 822

${ }^{17}$ Patrick W, Webster K, Ludwig L, Roberts D, Wiebe P, Younes M. Noninvasive positive-pressure ventilation in acute respiratory distress without prior respiratory failure. Am J Respir Crit Care Med 1996; 153: 179 - 193

${ }^{18}$ Hoffmann B, Welte T. The use of noninvasive pressure support ventilation for severe respiratory insufficiency due to pulmonary oedema. Intensive Care Med 1999; 25: 15 - 20

${ }^{19}$ Wiener CM, Kirk W, Albert RK. Prone position reverses gravitational distribution of perfusion in dog lungs with oleic acidinduced lung injury. J Appl Physiol 1990; 68: 1386 - 1392

${ }^{20}$ Ambrosino M, Foglio K, Rubini F, Clini E, Nava S, Vitaca M. Noninvasive mechanical ventilation in acute respiratory failure due to chronic obstructive airway disease: correlates for success. Thorax 1990; 50: 755 - 759

${ }^{21}$ Bott J, Carroll MP, Conway H. Randomized controlled trial of nasal ventilation for hypercapnic respiratory failure in chronic obstructive pulmonary disease. Lancet 1993; 341: 1555 - 1557

${ }^{22}$ Kramer N, Meyer TJ, Meharg J, Cece RD, Hill NS. Randomized, prospective trial of noninvasive positive pressure ventilation in acute respiratory failure. Am J Respir Crit Care Med 1995; 151 : 1799 - 1806

${ }^{23}$ Laier-Groeneveld G, Kupfer J, Hüttemann U, Criee C-P. Weaning from invasive mechanical ventilation. Am Rev Respir Dis 1992; 145: A518

${ }^{24}$ Udwadia ZF, Santis GK, Steven MH, Simonds AK. Nasal ventilation to facilitate weaning in patients with chronic respiratory insufficiency. Thorax 1992; 47: 715 - 718

${ }^{25}$ Restrick LJ, Scott AD, Ward EM, Feneck RO, Cornwell WE, Wedzicha JA. Nasal intermittent positive-pressure ventilation in weaning intubated patients with chronic respiratory disease from assisted intermittent positive-pressure ventilation. Respir Med 1993; 87: 199 - 204

${ }^{26}$ Nava S, Ambrosino N, Clini E, Prato M, Orlando G, Vitacca M, Brigada P, Frachia C, Rubini F. Noninvasive mechanical ventilation in the weaning of patients with respiratory failure due to chronic obstructive pulmonary disease. Ann Intern Med 1998; 128: $721-728$

${ }^{27}$ Girault C, Daudenthun I, Chevron V, Tamion F, Leroy J, Bonmarchand G. Noninvasive ventilation as a systematic extubation and weaning technique in acute-on-chronic respiratory failure. A prospective, randomized controlled study. Am J Resp Crit Care Med 1999; 160: 86 - 92

${ }^{28}$ Nourdine K, Combes P, Carton MJ, Beuret P, Connanda A, Cucreux $\mathrm{J}-\mathrm{C}$. Does noninvasive ventilation reduce the ICU nosocomial infection risk? A prospective clinical survey. Intensive Care Med 1999; 25: $567-573$

${ }^{29}$ Kollef MH. Avoidance of tracheal intubation as a strategy to prevent ventilator-associated pneumonia. Intensive Care Med 1999; 25: 553 - 555

${ }^{30}$ Laier-Groeneveld G, Criee C-P. Noninvasive mechanical ventilation in weaning from mechanical ventilation. Eur Respir Mon 1998; 8: 299 - 327

${ }^{31}$ Marini JJ. Should PEEP be used in airway obstruction? Am Rev Respir Dis 1989; 40: 1 - 3

Prof. Dr. med. Joachim Lorenz

Abteilung für Pneumologie, Infektiologie, Intensivmedizin und Schlafmedizin

Kreiskrankenhaus Lüdenscheid

Postfach

58505 Lüdenscheid 